\title{
MATEMÁTICAS Y LÓGICA: PROFESORES, AUTORES, PROGRAMAS Y MANUALES EN LA ENSEÑANZA SECUNDARIA DE LAS ISLAS CANARIAS DURANTE LA SEGUNDA MITAD DEL SIGLO XIX
}

\author{
Juan Francisco MarTín del CASTILlo \\ I.E.S. Francisco Hernández Monzón \\ (Las Palmas de Gran Canaria)
}

RESUMEN. Las Matemáticas y la Lógica Informal son los dos asuntos a tratar en el presente trabajo. A través del profesorado, sus programas de asignatura, así como los manuales, si los hubiere, es como se trata de averiguar cuáles eran las líneas del currículo impartido en la segunda mitad del siglo XIX, precisamente cuando en las Islas Canarias se extiende, al menos en términos relativos, el proceso educativo en una manera jamás vista antes. El objetivo principal de la investigación es poner al descubierto un capítulo desconocido de la historia contemporánea de la educación en España, además de presentar algunas características propias y diferenciales de la Segunda Enseńanza en este rincón del Atlántico, que no siempre coinciden con el grueso histórico del sector en el país.

Palabras ClaVe. Enseñanza Secundaria, Matemáticas elementales (Aritmética), Lógica informal, Profesores, Programas, Manuales, Islas Canarias, Siglos XIX-XX.

ABSTRACT. This article treats upon the Secondary Schooling in the Canary Islands (Spain) during the second half of the Nineteenth Century. Mathematics and Informal Logic are the subjects to investigate. For instance, we will know what the most important short handbooks on Elemental Mathematics (Arithmetic) were and who their authors were. 
Across teachers, textbooks and programmes we will have the opportunity to understand what the summary and level of those logical lessons were. Finally my claim is to discover one of the unknown chapters of contemporary european, as well as spanish, History of Education.

KEYwORDS. Secondary Schooling, Elemental Mathematics (Arithmetic), Informal Logic, Teachers, Programmes, Handbooks, Canary Islands (Spain), XIX-XX Centuries.

\section{Introducción}

El presente trabajo versa sobre la Enseñanza Secundaria en las Islas Canarias durante la segunda mitad del siglo XIX, aunque pueda extenderse, en alguna manera, a los comienzos de la centuria entrante, donde no parece modificarse en demasía lo descrito para el tiempo anterior. En especial, las matemáticas y la lógica informal, pues no había posibilidad todavía a una alternativa, son los temas centrales de debate y exposición. Curiosamente, la historia de la educación en los puntos de la periferia española, y aún europea, ha sido merecedora de escasa atención, por no decir algo peor, cuando lo que se imponía era la visión opuesta: descubrir, analizar y comparar modelos de ambos lados para, una vez contextualizados, extraer las debidas conclusiones. A buen seguro, ello habría favorecido en grado sumo el conocimiento y difusión de las disposiciones educativas emanadas de las autoridades gubernativas $y$, en coincidencia con este punto, la diversa lectura que se hizo de ellas en lugares distantes del mapa nacional.

Realmente, los temas, aquí estudiados, son muy bien conocidos en la mayoría de los países de nuestro entorno europeo inmediato. $\mathrm{O}$, al menos, y por introducir un tono de moderación en la anterior afirmación, han recibido un adecuado tratamiento histórico. Sin embargo, en España resta mucho por hacer, si bien ya se han dado pasos decididos en la dirección apuntada, con el estudio de los manuales de instrucción, o catecismos como se gustaba decir en la época, de las diferentes asignaturas de los programas educativos, centrándose en las listas aprobadas por el Ministerio que, de manera periódica, las hacía saber al profesorado. En las Islas Canarias, el sentimiento de vacío se palpa, por cuanto la historia de la educación adolece de un secular seguimiento parcial, incompleto a todas luces, como lo ha reconocido uno de los especialistas más autorizados de 
la temática ${ }^{1}$. Las investigaciones, aunque no globales ni en el tiempo examinado ni en el asunto tratado, informan de aspectos concordantes con la educación española en su conjunto, no obstante dejan grandes lagunas por explorar. Nuestra intención, sabido esto, es aportar luz sobre algunos capítulos de esta inacabada historia. Justamente, aquellos que están más olvidados o que, como es el caso, ni siquiera han sido atendidos por indiferencia o despreocupación.

Antes de comenzar en el ahondamiento temático, se hace perentoria una breve presentación de la situación educativa de las Islas Canarias en el siglo XIX. De hecho, son tres las características que reinan en el período: i) la fragmentación del territorio; ii) la ausencia de escuelas, o centros educacionales, como reflejo de un mal endémico en la historia insular; y iii) el altísimo índice de analfabetismo. Devienen en esenciales cuando lo que se desea es comprender la naturaleza de los problemas de la educación en Canarias y, de modo especial, la Segunda Enseñanza.

\section{i) Territorio fragmentado}

Es difícil obtener una completa visión de esta cuestión si no se tiene un ánimo de empatía con el problema. Por supuesto, para el nativo es relativamente fácil explicar lo que produce vivir en islas y los serios obstáculos que llegan a presentarse, en determinadas circunstancias, con motivo de los desplazamientos forzados. Uno de estos impedimentos es, precisamente, el fluido desarrollo del proceso educativo para un joven isleño de la época, que, por necesidad, no le quedaba otro remedio que acudir a centros alejados de su tierra natal, si habitaba en las islas más alejadas, para poder responder ante los tribunales pertinentes de su competencia a través de los exámenes de reválida. Si a esto último, unimos el ansiado acceso universitario, la aventura tomaba cariz de auténtica odisea porque el viaje podría llevarle más allá de los Pirineos, como así fue en el caso mayoritario de los médicos, formados en Francia o Escocia. Queda por decir, aunque vaya implícito en el discurso, que los afortunados que lograban promocionar en tal sistema provenían, sin excepciones, de las clases acomodadas, las únicas en condiciones de afrontar semejante gasto, además de disponer a su gusto de estas oportunidades.

\footnotetext{
'Véase Negrín [1982, passim].
} 


\section{ii) La ausencia de escuelas}

Esta característica es importante desde el punto de vista del número de posibilidades efectivas para educar a los jóvenes. Las primeras letras, o el nivel más bajo, sólo estaba disponible en algunas de las islas, habida cuenta la falta de profesorado que llevara adelante la tarea. En efecto, aquellas ciudades que contaran con compañías de regulares (la orden de los Jesuitas, por ejemplo [ESCRIBANO, 1987]), serían las favorecidas con el regalo educativo, porque, entre los objetivos de los religiosos, estaba el impartir docencia. También algunas sociedades filantrópicas, y en punto coincidente con el sesgo nacional, ayudarán en semejante apuesta, destacando las Reales Sociedades Económicas de Amigos del País, interesadas en la mejora de la población en todos los sentidos. Sin embargo, y pese al entusiasmo de unos y otros, la ausencia de escuelas y centros educativos en general es una evidencia constante e incontestable de la historia de la educación en las Islas hasta las primeras décadas del siglo XX.

\section{iii) El analfabetismo}

El alto índice de analfabetismo en Canarias es un problema endémico ${ }^{2}$. Como es bien conocido, andaluces y canarios son los que, históricamente, mayores cotas han alcanzado en España. Por descontado, este fenómeno está ligado a la paupérrima situación social, a la cual, en cierta manera, deja al descubierto. Quiere decirse que los niveles de empobrecimiento socioeducativo espejean a la sociedad en la que se originan. Hay que resaltar, por ende, que se faltaría a la verdad histórica si no se atendiera a este principio fundamental a la hora de someter a análisis cualquier aspecto de la historia educativa so pena de vernos en un discurso vacuo e irresponsable con los agentes históricos.

En fin, estos son los tres hitos que, irremediablemente, hay que tener en la mente cuando se pretende contextualizar la educación en Canarias y, más todavía, en el tiempo que nos hemos marcado como marco de referencia, aunque se hayan dado avances significativos con respecto a centurias pasadas. Visto, de manera somera, el contexto, lo justo es presentar el asunto a estudiar. p. 8].

${ }^{2}$ El 80 por ciento de la población no sabla leer ni escribir. Véase SUÁrEz FaLCON [1920, 


\section{Matemáticas: autores y manuales}

Domingo J. Navarro, doctor en Medicina y cronista verídico de los avatares de la ciudad de Las Palmas de Gran Canaria, describió como eran las enseñanzas de las ciencias en el tiempo que le tocó vivir y, especialmente, de las matemáticas a comienzos del siglo XIX. Juzga en términos negativos la situación, vale decir incluso que de forma tajante: «las matemáticas apenas saludaban el álgebrai [NAVARRO, 19991, p. 74]. Sin embargo, este perfil frustrante nos ha de servir de contraste con la perspectiva de la educación en las Islas Canarias después de 1890. En tal sentido, el propio Navarro se alza en testigo y juez a un mismo tiempo. Primeramente, como estudiante de un sistema en declive y, más tarde, como profesor en uno de los más importantes centros educativos del archipiélago y radicado en la capital citada: el Colegio de San Agustín ${ }^{3}$.

En pocas palabras, las matemáticas no gozaban, cuando menos en el sector mayoritario de las escuelas o colegios, de profesores lo suficientemente preparados en la materia. En mayor perjuicio todavia, el modelo educativo, recién impuesto, no fue recibido entre alborozos, ya que exigía una calidad profesional, por parte del docente, que lejos estaba de ser alcanzada en el período. Por esta razón, los manuales, ya fueran de autor español o directa traducción de los originales franceses - lo más habitual-, no se encontrarán en los depósitos de los museos históricos, como, por ejemplo, el Museo Canario. Este dato no debe caer en ningún momento en el olvido, ya que, superada la natural extrañeza, hará brotar la reflexión acerca de quiénes, en consecuencia, son los hacedores de los catecismos publicados y difundidos en las Islas, y cuál es, en definitiva, la motivación de la impresión de los pequeños manuales. Finalmente, y como apunte discordante con lo anterior, la mayoría del estudiantado no optaba por las áreas científicas, en sus deseos de proseguir la carrera universitaria, máxime el nulo valor utilitario de semejante expectativa.

\subsection{4-1900: primeros desarrollos}

El curso 1844-1845 es muy importante en la Historia de la Educación en las Islas Canarias. En este año, y es solamente un aviso, la sociedad civil acepta como un reto las medidas de promoción educativa dispuestas desde Madrid. Así, en

${ }^{3}$ El colegio abrió sus puertas en 1845 por Real Orden de 23 de marzo. 
1846, por Real Orden de 21 de agosto, es abierto a la matrícula del alumnado el Instituto Provincial [FAJARDO, 1995], en la isla vecina de Tenerife, único con tal rango en el conjunto insular durante mucho tiempo, hasta que en 1916 se cree otro centro en Gran Canaria de igual categoria y reconocimiento oficial.

El espíritu de cambio, además de la reforma de los curricula, hará aparecer un nuevo modelo. Durante los próximos años, y dentro del período democrático consiguiente a la Gloriosa (1868-1874), las disposiciones promoverán que los situación, al menos en la superficie, se tambalee y prevalezca un cierto aire de novedad e intencionalidad progresista. No obstante, el movimiento de mejora en las Islas Canarias es bastante diferente al resto del Estado. Las causas de este fenómeno ya han sido definidas con antelación. Por lo tanto, el modelo canario, si existiera tal cosa en sí, viene a ser una peculiar visión del esfuerzo reformista. Lo que sigue es buena prueba del aserto recién realizado.

Una vez revisada la historia local de la Segunda Enseñanza, y comprendido el fenómeno, estamos listos para afrontar la realidad de los libros escolares, cuyos autores fueran canarios o no. En 1849, se edita el primer catecismo, teniendo como hilo argumental la exposición de las matemáticas elementales (Aritmética de niños, arreglada para el uso de las escuelas), pero sin que constara firma de autor por ninguna parte, a excepción de un acrónimo que nada informaba de la responsabilidad de la escritura: D.B.C.B. Este librito abre, si se nos permite la expresión, el mundo de la edición a los jóvenes profesores preocupados por el nivel educativo de su comunidad. Uno de ellos fue Juan de la Puerta Canseco, principal protagonista del evento y que, con el devenir de los años, se erigirá en adalid de los manuales escolares en las Islas ${ }^{4}$.

Puerta Canseco es el autor por excelencia de estos textos de matemáticas en el archipiélago. Lo dicho no es una exageración gratuita sino una evidencia histórica. Sus trabajos fueron publicándose a lo largo de la segunda mitad del siglo XIX, copando, en determinados instantes con tiradas de 500 ó 2000 ejemplares, el mercado de edición de textos de escolaridad. Por ejemplo, el Compendio de Aritmética, que gozó nada menos que de quince ediciones en un intervalo relativamente corto (1857-1892), es una obra a la que nadie le discute el valor peda-

4 Véase MARTIN DEL CASTILlo [1997] y [1999]. Sobre el mundo de las imprentas insulares, cfr. VizCAYA [1964], LUXÁN [1994] y COLA BENITEZ [2001]. 
gógico y, por supuesto, histórico. Por desgracia, la personalidad de Puerta Canseco, salvo algunos detalles aportados por el cronista Isaac de Viera en Vidas ajenas (1888), es desconocida para los modernos historiadores. Antes del Compendio, redactó y dio a la estampa un interesante opúsculo, Nuevo sistema legal de medidas, pesas y monedas (1852), que supuso su primer gran éxito en el mundo editorial $^{5}$, animándole para ulteriores aventuras. A todas estas, el objetivo del publicista no era otro que ofrecer a los estudiantes unos textos didácticos, por lo menos, para la época y que también habrían de servir de recordatorio, en el caso de los lectores adultos, de las enseńanzas dejadas atrás. La difusión de la "Primera Parte" del Compendio de Aritmética, en 1857, pues realmente la obra estaba proyectada con tres apartados, impresos separadamente, según reza en la advertencia preliminar del volumen, marcó un hito en la Historia de los Manuales Escolares de Canarias. De hecho, Puerta Canseco fue pionero en relación a la edición de los programas de las asignaturas dentro del contexto insular. Estuvo en la vanguardia en todos los sentidos, desde los propósitos didácticos hasta la esfera política, donde se granjeó una merecida fama de entusiasta reformador de los ideales de la educación ${ }^{6}$.

La Cartilla comercial (1870) es otro de sus textos que figuran con letra de molde en la historia educativa. Diseñada para explicar, detallar y dar consejo sobre la contabilidad de libros y, de modo generalizado, acerca de los «estudios de aplicación», nacidos de la Ley Moyano de 1857, tuvo su referente inicial en el Compendio, toda vez que se anticipaba en sus líneas el contenido de la Cartilla. Si bien no llegó a reproducir los índices de tirada de su antecesor, puede decirse, sin temor a equivocarse, que el impacto popular que promedió la nueva obra ratifican el puesto de Puerta Canseco como exitoso escritor de catecismos escolares.

El último tercio del siglo XIX refleja un balance positivo en el ritmo de las publicaciones de carácter didáctico, seguramente como respuesta al boom del leonés. En la actualidad, prosigue el debate historiográfico sobre algunas de las incógnitas que suscitan las ediciones de Puerta Canseco. La más acusada, y difícil de resolver, ya que no hay material documental que pruebe una teoría u otra a ple-

5 Sobre la introducción del nuevo sistema métrico decimal, véase la actualizada obra de AZNAR GARCla [1999].

${ }^{6}$ MarTín del Castillo [2000, pág. 467]. 
nitud, es el verídico aprovechamiento de los manuales publicados. Pues, es verdad innegable la difusión de las obras, pero lo que no está del todo claro es si el alumno, matriculado en el Instituto Provincial de Santa Cruz de Tenerife o en el oficioso Instituto de Las Palmas, utilizaba a diario esa herramienta y, menos aún, si lo hacía en el aula. Esto último, según las averiguaciones recientes fundamentadas en los informes o memorias anuales de los centros, era más que improbable, puesto que, de la lectura atenta de ellos, se desprende que el profesorado de Matemáticas mantenía intactas las líneas programáticas del Ministerio, incluso respetando los autores y títulos recomendados. Esta realidad nos conduce de nuevo al principio, sin embargo queremos pensar que el lector adulto fue el principal consumidor del producto pedagógico. Para sostener este argumento, hay que echar mano de las notas características de la educación en Canarias y, especialmente, las dos últimas: la carencia de escuelas y los niveles de analfabetismo. Partiendo de ellas, cabe apuntar a un uso del Compendio o la Cartilla que no es el puramente académico, en sentido estricto, sino a una actualización de conocimientos o, en el caso más aventurado aunque no descabellado, al autodidactismo.

\subsection{Juan de la Puerta Canseco y su producción bibliográfica}

Nacido en Valencia de Don Juan, provincia de León, en 1827. Como quedó dicho, aparte de este dato y otros de índole editorial o social, poco más se sabe de él $\mathrm{l}^{7}$. Seguramente, arribó a las Islas Canarias después de haber tenido experiencias educativas en la capital del Estado, como maestro superior, en varias escuelas privadas. Conforme a lo editado por Isaac de Viera, fue el responsable directo, como cargo de la administración educativa isleña, de la creación de más de cincuenta colegios en el archipiélago. Activista decidido de la reforma, a través de múltiples cauces como las ediciones o los periódicos destinados a profesionales de la educación, residió la mayor parte de su vida en la capital santacrucera, donde dejó una huella indeleble entre los que le conocieron, además de la deuda que la sociedad canaria ha contraído con su obra. El dato y lugar de su muerte nos es desconocido por el momento.

La lista que sigue detalla la producción matemática de Puerta Canseco con toda la información necesaria para dar con ella. Nos parece, cuando menos, de

${ }^{7}$ Solamente las palabras de VIERA [1888, pp. 71-73]. 
obligada referencia, ya que, si bien lo antedicho ofrece una semblanza de lo conseguido históricamente, la propia fuente la reforzará como es debido ${ }^{8}$. Está desglosada por años, comenzando por 1852:

(1852) Nuevo sistema legal de medidas, pesas y monedas, precedido de unas breves nociones de aritmética decimal. Para uso de las escuelas de ámbos sexos. Santa Cruz de Tenerife: Imprenta y Librería Isleña, 47 páginas.

(1856) Nuevo sistema legal de medidas, pesos y monedas, precedido de unas breves nociones de aritmética decimal. Para uso de las escuelas de ámbos sexos. Santa Cruz de Tenerife: Imprenta de la Viuda e hijos de V. Bonnet, 2 ed.

(1857) Compendio de Aritmética para uso de las escuelas de ambos sexos. Primera Parte. Santa Cruz de Tenerife: Imprenta y Librería de la Viuda e hijos de D. V. Bonnet, 64 páginas.

(1859) Compendio de Aritmética - 2. ${ }^{a}$ Parte. Santa Cruz de Tenerife: Imprenta de Salvador Vidal, 52 páginas.

Compendio de aritmética para uso de las escuelas de ambos sexos. Tercera Parte. Santa Cruz de Tenerife: Imprenta de la Viuda e hijos de D. Vicente Bonnet, 48 páginas.

(1864) Problemas de aritmética. Cuaderno Primero. Santa Cruz de Tenerife: Imprenta de Salvador Vidal, 32 páginas.

Problemas de Aritmética. Segundo Cuaderno. Santa Cruz de Tenerife: Imp. Salvador Vidal, 32 páginas.

(1866) Compendio de Aritmética para uso de las escuelas primarias de uno y de otro sexo. (1. ${ }^{a}$ Parte. $4{ }^{a}{ }^{a}$ Edición). Santa Cruz de Tenerife: Imp. Y Librerra de José Benítez, 66 páginas +1 hoja.

(1868) Compendio de aritmética, 1. Parte. 5. Edición. Santa Cruz de Tenerife: Imp. y librería de J. Benítez, 66 páginas.

Compendio de aritmética, 3. Parte. 2." edición. Santa Cruz de Tenerife: Imp. y Librería de J. Benítez, 65 páginas. (Edición de 500 ejemplares).

${ }^{8}$ Naturalmente, esta producción debe ser puesta en relación con la exhibida en el resto del Estado. A ese fin, sería recomendable la lectura de VEA MUNIESA [1992], con la salvedad de que los fenómenos periféricos no son observados con el adecuado rigor ni por éste ni por ningún otro autor que aborde la temática de las matemáticas en la educación española de forma generalizada. 
(1870) Cartilla comercial para uso de los niños. Santa Cruz de Tenerife: Imp. y Librería de J. Benítez y C. ${ }^{2}, 74$ páginas.

(1874) Compendio de Aritmética. 1. ${ }^{a}$ Parte. 8. ${ }^{a}$ Edición. Santa Cruz de Tenerife: Imprenta y librería de J. Benítez. (Edición de 2000 ejemplares).

(1879) Compendio de Aritmética. 3. ${ }^{a}$ Parte. 3. ${ }^{a}$ Edición. Santa Cruz de Tenerife: Imprenta y Librería de J. Benítez y C. ${ }^{2}, 64$ páginas.

(1885) Cartilla comercial arreglada para uso de los niños, 3. Edición. Santa Cruz de Tenerife: Imprenta y Librería y Encuadernación de J. Benítez, 72 páginas.

(1892) Compendio de Aritmética para uso de las escuelas primarias. Santa Cruz de Tenerife: Imprenta de A. J. Benítez, 66 páginas. (Decimoquinta edición de la "Primera Parte» con 2000 ejemplares).

\section{Lógica: programas y profesores}

En este segundo bloque del trabajo, tomaremos cuenta del profesorado, amén de las programaciones y el marco legal en el que se movían, del área de Psicologia, Lógica y Filosofia Moral durante un período histórico ciertamente importante del desarrollo social y educativo del conjunto archipielágico, cual es el que arranca en 1870 y finaliza en 1911. La razón y motivo de esta parte es el encuentro de las grandes líneas fuerza que explican la evolución del concepto de lógica en aquel tiempo.

\subsection{Frente legal: 1857 y 1868}

La Ley de Instrucción Pública, de septiembre de $1857^{\circ}$, fue promulgada por el ministro Claudio Moyano con un criterio reformista con respecto a lo establecido. Las consideraciones sobre el fundamento y, sobre todo, las consecuencias de la ley son muchas, pero nuestro interés primordial está centrado en la comprensión del devenir curricular y, de modo preferente, en los temas relacionados con las asignaturas de perfil filosófico. La Segunda Enseñanza, conforme

9 Sobre los textos legales, es de consulta casi obligada UTANDE [1964], aunque el mismo VEA MUNIESA [1992, II, 685ss. (apéndice)] ofrece un listado exhaustivo. 
al nuevo referente legal, estaba dividida en dos: por un lado, una etapa de Estudios Generales, y, del otro, lo que es más interesante, una fase de los denominados Estudios de aplicación a las profesiones industriales. En realidad, esta separación era un buen intento por renovar el mundo de la educación, aunque, desafortunadamente, la práctica diaria borró cualquier atisbo de optimismo sobre la bondad de los principios de la nueva ley.

El hecho es que los Estudios Generales son el centro de nuestro examen. Se subdividían en dos fases de 2 y 4 años respectivamente, pero lo relevante, jurídicamente hablando, reside en el contenido del artículo número 15 del cuerpo legal, que define el currículo para la segunda de las fases citadas:

\section{- Religión y Moral cristiana.}

- Ejercicios de análisis, traducción y composición latina y castellana.

- Rudimentos de lengua griega.

- Retórica y Poética.

- Elementos de Historia Natural y de la particular de España.

- Ampliación de los elementos de Geografía.

- Elementos de Aritmética, Álgebra y Geometría.

- Elementos de Física y Química.

- Elementos de Historia Natural.

- Elementos de Psicología y Lógica.

- Lenguas vivas.

Las dos asignaturas en cursiva son las que representaban, con sus propios programas, a las áreas afectas a la consideración filosófica. Como se puede apreciar, hay una clara delimitación de lo que es el aspecto normativo con referencia al aspecto epistemológico, por decirlo en la terminología en boga. En reducida cuenta, la Ley Moyano hacía distinción entre lo que estaba destinado a la formación del espíritu y actitudes del estudiante de secundaria, abriendo una zanja curricular con las lecciones de introducción a lo que siempre había sido el pensamiento filosófico. Más tarde, no obstante, ambas se unificarán en un solo bloque de conocimientos, pero, para que ello fuera factible, debió de intermediar un 
período revolucionario. La ley de 1857 , en suma, no consiguió colmar los objetivos propuestos en un comienzo, pues, al decir incluso de algunas voces contemporáneas, se perdió una gran oportunidad de renovar el campo educativo por la ausencia de concreción de determinadas medidas o, dicho en forma más concisa, por la irrealidad en que se fraguaron la mayor parte de sus principios de ejecución. Un ejemplo bastará para hacer entendimiento. No hubo una distribución de horas por áreas o asignaturas, fijada de antemano por el legislador, amén de adolecer de una carencia casi total de planificación semanal de los estudios. En fin, no existió un criterio serio de aplicación de la reforma legal, que, contrariamente, sí aparecerá en sucesivas disposiciones.

El Plan de Estudios de octubre de $1868^{10}$ se mostrará diferente en un todo. No sólo concretará el esquema de horas y asignaturas, sino que el currículo también se verá afectado como resultado del impacto de las ideas liberales y progresistas. De nuevo, la Enseñanza Secundaria queda partida en dos momentos: estudios con o sin el latín. La intención del legislador era respetar, en lo mínimo, todas las facciones sociales del pueblo español, incluida la anticlerical, en un afán de adaptarse a los nuevos tiempos y creencias. Por supuesto, la reforma dependía, en grado extremo, de la situación política, pero no tanto como de la voluntad individual del profesorado por llevar adelante el desarrollo del novedoso diseño. Los estudios "con latín", como era de esperar, fueron los que obtuvieron la mayor tajada educativa a causa de la inveterada tradición del sector católico en el entramado social. Supuestamente, la presencia del latinismo habría dado un giro conservador al espíritu reformista, más que contradictorio con su ideario de partida, pero no fue así porque el voluntarismo docente, aun respetando los deseos de la mayoría, pudo enseñar el nuevo currículo. Éste quedará fijado del modo próximo:

- Gramática latina y castellana (primer curso) - diaria.

- Gramática latina y castellana (segundo curso) - diaria.

- Elementos de Retórica y Poética - diaria.

- Nociones de Geografía - alternante.

- Historia de España - alternante.

${ }^{10}$ Vea Muniesa [1992, II, 517ss.]. 
- Aritmética y Álgebra - diaria.

- Geometría y Trigonometría (rectilínea) - diaria.

- Elementos de Física y Química - diaria.

- Nociones de Historia Natural - alternante.

- Psicologia, Lógica y Filosofia moral - diaria.

- Fisiología e Higiene - alternante.

Bien se ve, lo que antes eran dos, ahora ha quedado reducido a un solo elemento curricular, específicamente definido como una introducción a la Filosofia, que en nada recuerda la exigente separación de 1857. De otra parte, el aspecto religioso no entra, al menos en la previsión inicial, en los planteamientos propuestos. Con todo, a este respecto, la realidad local, en cada centro de las regiones españolas, marcará la evolución del Plan de Estudios.

\subsection{Profesores de Lógica en Canarias (1870-1911)}

Hasta 1927 las Islas Canarias serán una única unidad administrativa. Esta provincia disponía, a su vez, de un solo instituto oficial en Santa Cruz de Tenerife pero, de hecho, hubo dos centros en el archipiélago: 1) el Instituto Provincial, ya mencionado y reconocido como tal, y 2) el irregular Instituto de Gran Canaria o Las Palmas. Lo que debe tenerse presente, en todo instante, es que ambos son las únicas fuentes seguras para examinar las lecciones de lógica en aquel período. Por medio de las memorias anuales, editadas poco después de la entrada en vigor del anterior Plan, es posible hacerse una imagen fidedigna, aunque tamizada por la dirección del centro, de los hechos relativos a la Segunda Enseñanza en las Islas, desde los profesores del claustro hasta los manuales y asignaturas.

\section{a) El Instituto Provincial (1868-1911)}

La Memoria de 1869 [PINTO, 1870] indica al Dr. Francisco Rodríguez de la Sierra como el profesor a cargo del área, pero, por desgracia, no informa del libro o manual obligatorio que servía de referente. El siguiente informe anual (1870) presenta la misma situación [PINTO, 1871], es decir, escasa, por no decir nula, información sobre el detalle de los contenidos programáticos de las lecciones 
impartidas por el docente. Ello habría de cambiar por un hecho lamentable como fue la muerte del profesor. Así, su sustituto, nombrado de forma interina por el cuerpo docente, hasta la nueva adjudicación por concurso público de la plaza, será un clérigo, y bastante reputado en la Isla por cierto. La designación tuvo plena efectividad en febrero de 1870 y se consideraron como méritos, más que suficientes para desempeñar el puesto, la acreditada categoría profesional del candidato, a la sazón profesor de Teología, así como su posición entre el clero local, puesto que ostentaba la dignidad de Canónigo Doctoral.

El que así hacía valer su crédito no era otro que el Dr. Silverio Alonso del Castillo, que fue responsable de la asignatura por un período relativamente largo, habidas las circunstancias, pero de lo más interesante dentro del contexto socioeducativo (1871-1875). Como su antecesor, Alonso jamás explicó cuáles eran sus orientaciones en cuanto a los programas y menos aún sobre la posibilidad de un texto de mano. Sin embargo, este secretismo didáctico es reflejo claro de las prebendas del Plan de Estudios de 1868, que, en contadas ocasiones, como ésta, jugaban en su propia contra. Porque el eclesiástico no deseaba hacer públicas sus preferencias, a ciencia cierta de corte conservador, y así provocar la airada respuesta de los sectores menos afines del claustro o de la sociedad civil. No le importaba, en suma, el silencio sobre el programa ya que, entre otras cosas, la normativa se lo permitía. Con todo, se puede rastrear el pensamiento de Alonso del Castillo en medio de algunos de sus discursos leídos en público y en atribución de sus facultades como Canónigo Doctoral. De hecho, llegó a pronunciar las siguientes palabras, elocuentes por sí mismas: «(...) lógica de Aristóteles, la que nadie ha podido echar por tierra porque es el camino real por donde sin tropiezo marcha siempre la razón»" ${ }^{11}$. La mención del Estagirita deja, en transparencia, cuál era el sentido que daba el clérigo a sus clases. Para él, al igual que para muchos de su época, la lógica comenzaba y terminaba en los trabajos de Aristóteles. Por supuesto, Francis Bacon e Inmanuel Kant eran por completo desconocidos en estos menesteres: su Nueva Lógica no estaba dentro del canon eclesiástico.

El curso de 1875/76 representa una importante inflexión en la historia escolar del área. El profesor Agustín Arredondo y García, proveniente de Granada,

$"$ Alonso del. Castillo $[1881$, p. 13]. 
entra a formar parte del Instituto Provincial, por haber superado los exámenes oficiales, convocados y celebrados en la Universidad Central de Madrid, que le habilitaban como docente cualificado de la asignatura ${ }^{12}$. Esto es, inaugura una nueva etapa que, salvada la interinidad del puesto, se caracterizará por una decidida apuesta por la claridad y suficiencia de los contenidos, además de enfatizar la solemnidad de la asignatura en el marco del renovado currículo. Estuvo, al menos, tres años en las Islas Canarias y dejó, huelga decirlo, honda huella en la institución no menos que en la historiografía local, pese a lo reducido de la estancia.

Como se ha insinuado el destino laboral fue Tenerife, el primero de una larga y bregada carrera profesional, y enseguida tomó el pulso a la situación académica. De inmediato, tanto que sorprende, escribió y editó varios textos relativos a las asignaturas de su responsabilidad. En 1875, por ejemplo, publicó el Compendio doctrinal de Psicologia, Lógica y Filosofia Moral. (1. ${ }^{2}$ Parte), aunque antes ya había dado a la imprenta el programa completo del área [ARREDONDO, 1875a]. $\mathrm{Al}$ año siguiente, completó el Compendio con la segunda parte, dedicada en exclusividad a la exposición de las lecciones de lógica. En determinada manera, cabe decir que Arredondo comparte con Puerta Canseco el honor de ser pionero en la edición de catecismos, o pequeńos manuales didácticos, ya fuera de la Lógica, en un caso, o de las Matemáticas básicas, en el otro, dentro del contexto canario de la Restauración.

Las intenciones, amén de las formas, de Arredondo están plenamente integradas en el espíritu del Plan de Estudios. Como joven docente, experto en su área de trabajo y recién aprobado en las oposiciones, no le duelen prendas en hacer públicos la orientación y contenidos de los programas explicados en el aula. Más bien, todo lo contrario. A más de esto, hacía gala de una personalidad liberal, perfectamente adaptada a los principios septembristas. No obstante, la continuidad de la tarea emprendida en las Islas fue interrumpida por su íntimo deseo de regresar a tierras andaluzas. En la Memoria de 1878/79 queda patente la noticia de su traslado a Andalucía:

«Anunciado el concurso para la provisión de la Cátedra de Psicología, Lógica y Filosofía Moral del Instituto de Almería, fue trasladado á ella por

12 BENITO [1876, p. 13]. 
Real Orden de 11 de Marzo de este año nuestro digno compañero el Lido. D. Agustín Arredondo y García, el cual tomo posesión de nuevo cargo el 5 de Abril siguienten. ${ }^{13}$

Arredondo continuó, como era previsible, con su actividad en favor de la edición de libros didácticos para el aula. Verbigracia, publicó en 1881 el Tratado de Psicología, Lógica y Ética. A pesar de la contrariedad del traslado, ello no supuso el cese de las funciones dentro del instituto, en el área específica del docente granadino. El Licenciado Antonio Zerolo desempeñará el cometido, mostrándose fiel en todo momento a las directrices heredadas, compartiendo incluso el programa impreso por el andaluz. Mas, el informe de 1879/80 describe un especial acontecimiento que, quiérase o no, influirá decisivamente en el currículo ${ }^{14}$. Dos profesores intercambian, por permuta, sus destinos profesionales. Vicente Rodríguez García, el que nos interesa, arriba a Santa Cruz de Tenerife en calidad de catedrático, deshaciendo anteriores componendas del claustro en satisfacción de las necesidades del alumnado y, cómo no, acatando la normativa vigente. El nuevo profesor, muy experimentado, pues no en vano su carrera se había iniciado en 1846 , conectará con viejas posturas, más propias de la primera mitad de la centuria. Una de las notas que delatará su perfil curricular es la imposición del volumen de Ceferino González, bastante conocido en el tiempo, y cuya orientación era latinista, como lectura obligada.

En 1882, Rodríguez y García fallece, con lo que la necesidad de cubrir la plaza vacante, en el menor tiempo posible, se hace patente de nuevo. Por esta razón, Francisco María Pinto de la Rosa, en un primer período (1882-1885), y Felipe de la Garza y Martínez, en un segundo (1885-1889), se hacen cargo de la responsabilidad, según consta en los papeles del Instituto [FAJARDO, 1995, 205206]. Ambos continuarán la línea dibujada por el antecesor, sin cambios en lo profundo. Sin embargo, Francisco Ruiz Macías marcará distancias con los planteamientos expuestos. Docente de varias asignaturas, algunas no conectadas directamente entre sí, pero todas ellas acordes con la planificación de los nuevos estudios, redactó un libro sobre lógica, que merece ser recordado. Su Compendio de Lógica (1904), de 188 páginas, no incluido por cierto en la magna Bibliografia

\footnotetext{
${ }^{13}$ ReYMUNDO [1879, pp. 45-46].

14 Alonso del Castillo [1882, pp. 27-28].
} 
Filosofica Hispánica (1901-1970) de Díaz y Santos [ver la bibliografía final], reclama nuestra atención. Para este autor, y es lo curioso, Francis Bacon es el auténtico renovador de la lógica en la historia de la materia, en sentido opuesto a la mayoría de sus colegas de profesión. Ocupará las labores de profesor de la asignatura, que luego se dividiría en dos en los sucesivos ordenamientos, hasta 1911.

b) El caso de Rafael Lorenzo y García en Las Palmas de Gran Canaria

Miguel de la Rosa, responsable de la Memoria letda el día $1^{\circ}$ de octubre, en la inauguración del curso académico de 1869 a 1870, en el Instituto de Segunda Enseñanza de Las Palmas de Gran-Canaria (Imprenta de la Verdad, 1869), escribió que Rafael Lorenzo García era el profesor de la asignatura «Psicología, Lógica y Ética». También informaba, de paso, que los libros de texto, mayormente usados en el mundo escolar, eran los de Monlau y Heredia.

Como presentación de Lorenzo, cabe referir que estaba considerado como un abogado de prestigio y solvencia acreditada, conforme a la imagen popular que se extendió de él, además de ensayista librepensador y redactor de varios libros acerca de la teoría de Darwin ${ }^{15}$. Realmente, al decir de Viera (1888, pp. 63-64), era un tipo asaz curioso, moteado de extrañas virtudes pero de una privilegiada inteligencia, fiel representación de la íntima brega por estar a la última en cuanto al conocimiento de las novedades editoriales, fueran de España o más allá. Nuestro propósito, sobra decirlo, es mostrar su pensamiento didáctico, si lo tenía, de la misma manera que desentrañar la índole de sus clases de lógica. En este aspecto, no tiene incomodo en hacer público el contenido de las lecciones, ya que publicó el programa del área en 1869, haciendo reedición en 1871. Esto es, en ningún momento quiso hacerse el esquivo ante la exigencia de los contenidos y lecturas a sugerir. Ello viene explicado por su misma conducta, atenta a la noticia reciente sobre la enseñanza en general y, en específico, sobre los manuales de Filosofía, de los cuales se declara auténtico devoto. Conoció y leyó las más importantes obras de los filósofos consagrados, amén de lo que le llegaba a las manos por petición directa a las casas editoriales - algo normal para el tiempo y el lugar-, y, en definitiva, pudo haber degustado de la lectura, en la lengua

\footnotetext{
15 Véase GLICK [1982, pp. 33s.] y SÁNCHEZ \& PAZ [1988, pp. 59-61].
} 
original, de los libros de Darwin y Feuerbach. Su postura, en lo relativo a la educación y los problemas generados por la planificación de asignaturas y modelos alternativos, es diferente a la del resto de sus compañeros. Esta disimilitud de posiciones, quizá, sea mejor vista a través de sus propias palabras, aunque el tema sea el programa de "Psicología, Lógica y Filosofía Moral»;

"(...) Por esto hemos reformado nuestro primer programa. Siempre hay que aprender y refutar. La escuela pedagógica del Dr. Buneke nos ha suministrado nuevos trabajos analíticos en psicologia, y los escritos de los nuevos materialistas alemanes, muy especialmente de Buchner y Feuerbach, nos han proporcionado motivo para aumentar las lecciones de la moral religiosa con refutaciones que no figuran en nuestro primer programan. ${ }^{16}$

Esta libertad académica de Lorenzo, entre otras cosas, demuestra la bondad del espíritu liberal de las disposiciones legales de la época. De no haber sido así, desde luego, que este profesor no hubiera resistido mucho tiempo en el empleo docente. Pero, no fue en absoluto así. Se mantuvo en el puesto bastante tiempo, no menos de cinco cursos completos. Aparece en las memorias finales de los años que cierran la década de los 60 y perdura, al menos, hasta el curso $73 / 74^{17}$, en que su nombre figura debajo del epígrafe que da cuenta del profesorado de la asignatura que le tocó ejercer durante tan largo período. Sin embargo, en la década entrante ello habrá de cambiar. El bachiller Pablo Padilla y Padilla será "uno" de los nuevos docentes, conforme a lo reflejado en la Memoria de 1881/82 [INGLOTT, 1881, p. 17]. No queda constancia, por cierto, del número y personalidad del resto. Y esta es toda la información disponible, mal que nos pese, sobre el Instituto de Las Palmas, puesto que los documentos conservados son escasos y fragmentarios en comparación con los de Santa Cruz de Tenerife, máxime cuando se trataba de un centro pseudooficial, mantenido por la tolerancia gubernamental. Aunque no podamos certificar, con total seguridad, que tras el paso de Lorenzo se replegaran a posicionamientos conservadores los sucesivos profesores del área en cuestión, sí que estamos en condiciones de asegurar que, en el proceso, las Islas no mostraron gran diferencia con respecto a la situación nacional. Y aquí, en verdad, si hubo un pliegue a los latinistas. Por ejemplo, Mariano

\footnotetext{
16 LORENZO [1871, página IV].

17 LUIS Y YAGUE [1874].
} 
del Amo y Águeda, profesor auxiliar en el Instituto de San Isidro de Madrid, primer centro de Segunda Enseñanza de la España contemporánea, publicó unos Elementos de Psicologia, Lógica y Ética seguin la doctrina de Santo Tomás (1885), que corroboran lo anterior, ya que este instituto actuaba como modelo a seguir por los demás del Estado.

\section{Conclusiones}

Sobre las Matemáticas hay tres puntos interesantes a resaltar:

a) Las matemáticas didácticas en las Islas Canarias fueron, durante el período examinado en el trabajo, idénticas al resto del conjunto estatal.

b) Sin embargo, la historia canaria de la Educación Secundaria es muy diferente a la nacional, especialmente en determinados puntos. Por esta razón, el balance de los contenidos matemáticos es similarmente diferente.

c) La edición de manuales o catecismos de Matemáticas Elementales (Aritmética) comienza en la segunda mitad del siglo XIX. El autor más relevante fue Juan la Puerta Canseco. Con él, las matemáticas escolares, al menos, disfrutaron de un período fértil de ediciones. Desgraciadamente, no nos es posible demostrar que sus libros fueran de uso común en las aulas de los dos institutos locales, porque la documentación no lo permite. En consuelo de esto, no puede dudarse del impacto popular de los trabajos del leonés, del que dan buena cuenta las tremendas tiradas de ejemplares con cifras escalofriantes para la época y el lugar.

Acerca de la Lógica podemos pronunciar similares palabras. La situación del profesorado de lógica o, por mejor decir, de la filosofía en conjunto resultaba curiosa y, por supuesto, interesante de todo punto. El Plan de Estudios de 1868 supuso un espaldarazo para aquellos docentes preocupados por adelantar novedades y conocimientos no presentes en los programas y manuales anteriores a aquél. Varios de ellos, entre los que cabe destacar a Agustín Arredondo, en Tenerife, y Rafael Lorenzo en Gran Canaria, se revelarán como ejemplos vivientes del nuevo espíritu pedagógico, no sólo en las clases diarias sino en los aspectos formales, al editar y difundir los contenidos programáticos de las asignaturas de su responsabilidad directa. Como final, la programación de la lógica, impartida en aquellos años, no difería en demasía del canon aristotélico, si bien algunos pro- 
fesores llegan a explicar a Bacon y Kant. Naturalmente, es una lógica informal, en absoluto la moderna lógica, en expresión de Carnap, que tardará mucho más tiempo en asentarse en España.

\section{Apéndice documental}

El texto, de más abajo, es el programa de lecciones sobre lógica de Lorenzo. (Apud LORENZO [1869, pp. 12-15]).

«57. Idea de la Lógica: tiene su base en la psicología: no es un arte, sino una ciencia, la ciencia del conocimiento.

58. Hácense de la lógica varias divisiones: Lógica natural ó popular y lógica artificial ó cientifica; lógica teórica y lógica práctica. Dícese también que hay una lógica pura y otra aplicada. Juicio que debe formarse de estas divisiones.

59. Utilidad de la lógica: su influencia como ciencia del conocimiento. La lógica constituye la educación del entendimiento: ventajas de una disciplina intelectual.

60. Definición de la crítica: su carácter analítico. Importancia del estudio crítico de nuestras facultades intelectuales.

61. Noción del juicio. Materia y forma de los juicios. Análisis del juicio bajo los aspectos psicológico, gramatical y lógico.

62. Formas lógicas de los juicios según la teoría de Kant: cantidad, cualidad, relación y modalidad.

63. Cantidad de los juicios: universales, particulares, singulares: ejemplos.

64. Cualidad de los juicios: afirmativos, negativos, indefinidos: ejemplos.

65. Relación de los juicios; categóricos, hipotéticos, disyuntivos: ejemplos. Modos de enlace en los juicios hipotéticos. Materia, forma y carácter de los juicios disyuntivos.

66. Modalidad de los juicios: problemáticos, asertóricos, apodícticos: ejemplos.

67. Motivos de juzgar. Noción de la certidumbre. No hay certidumbre sobrenatural. El carácter de la certidumbre es la evidencia, y ésta debe ser racional.

68. Noción de la evidencia: es objetiva y subjetiva. Probabilidad: su carácter variable. La duda: es afirmativa y negativa: indiferencia de la duda.

69. Definición del criterio: existencia de los criterios; sus clases los diversos orígenes.

70. Criterio de los hechos de conciencia: su legitimidad y sus límites. 
71. Criterio de los sentidos externos. Dificultad de la cuestión. Legitimidad de este género de certidumbre.

72. Criterio del testimonio humano: objeto del testimonio: es la fuente de un conocimiento doblemente sensible. Legitimidad de este conocimiento.

73. División del testimonio: es inmediato y mediato. Condiciones concernientes al testigo, veracidad, capacidad. Condiciones relativas al objeto, posibilidad y realidad del hecho. Condiciones referentes al que recibe el testimonio: arte hermenéutica: sus reglas capitales.

74. Criterio de la razón, sus motivos y sus legitimidad.

75. Criterio del sentido común: no es una facultad distinta del espíritu: su carácter de universalidad proviene de la razón común a todos los hombres. La universalidad no es la causa, sino el efecto, de la evidencia.

76. Noción de la dialéctica: su origen histórico. Según el pensamiento de Aristóteles, forma la dialéctica un parte integrante de la lógica.

77. Definición de la proposición: consta de tres elementos: dificultad de determinarlos en algunos casos, a consecuencia de la naturaleza de ciertas frases y de la índole de algunos idiomas.

78. Siendo la proposición el enunciado de un juicio por medio de la palabra, hay que considerar también en ella la cantidad, la cualidad, la relación y la modalidad.

79. División de las proposiciones por su materia: pueden ser incomplejas y complejas, simples y compuestas. ¿Mediante qué principios se reducen las proposiciones compuestas a simples?

80. Proposiciones idénticas y opuestas. Oposición coordinativa, subordinativa y diametral: proposiciones contrarias, subcontrarias, subalternas y contradictorias.

81. Noción del razonamiento. Diferencia entre el razonamiento y la demostración. Razonamiento deductivo e inductivo.

82. El razonamiento deductivo puede ser inmediato y mediato, de a dos y de a tres términos. Reglas generales del razonamiento deductivo: si las premisas son verdaderas, la conclusión es verdadera; si la conclusión es falsa, las premisas son falsas, pero no recíprocamente.

83. Definición del silogismo: su etimología. En el silogismo hay que considerar los términos y las proposiciones.

84. Clases de silogismos: categórico, hipotético y disyuntivo. Reglas del Silogismo categórico. 
85. Silogismos hipotéticos: principio de esta clase de razonamientos, su forma y su regla. 86. Silogismos disyuntivos: pueden formarse de a dos o más miembros: sus reglas. Combinación del silogismo hipotético y del silogismo disyuntivo: el dilema.

87. Argumentaciones no silogísticas: entimema, epiquerema, sorites, dilema y ejemplo.

88. Falacias: sus clases: el paralogismo y el sofisma: exposición de las más comunes. Medios de evitarlos y combatirlos.

89. Definición de metodologia: su importancia: orden que se ha de seguir en su estudio.

90. Noción del método: su división en analítico y sintético: Son independientes el uno del otro; pero deben unirse. Reglas generales del método.

91. Funciones integrales del método analítico: atención, observación, experimentación, generalización e hipótesis: esta es más bien auxiliar y supletoria.

92. La definición, La división, la clasificación y la demostración como funciones del método sintético: uso y relaciones de estas diversas formas científicas.

93. Noción de la definición: en qué se diferencia de la descripción. Definiciones experimentales y racionales. Reglas de la definición.

94. Idea de la división: a qué se aplica. Diferénciase del análisis. Reglas de la división. Co-divisiones, subdivisiones.

95. Noción de la clasificación: puede ser natural y artificial: ventajas de esta última para la determinación de las especies, principalmente en la botánica.

96. Noción de la demostración: es el complemento de las formas científicas del conocimiento. Contenido de la demostración. Demostración inmediata y mediata; directa e indirecta; a priori y a posteriori.

97. División del método, como medio de exposición de los conocimientos: es cientifico y popular, acroamático y erotemático.»

\section{Bibliografía}

Alonso del Castillo y Pérez, Mateo (1882): Discurso leido en el Instituto Provincial de Segunda Enseñanza de Canarias, en el acto solemne de la apertura del curso academico de 1880 á 81 ... y memoria del curso 1879 a 1880, por Quintín Benito y Benito. Santa Cruz de Tenerife, Imprenta de José Benítez.

AlONSO Del CASTILlo y PÉREZ, Silverio (1881): Discurso que en la solemne apertura del curso académico de 1881 a 82 leyó en el Seminario Conciliar de San Cristobal de La Laguna. Santa Cruz de Tenerife, Imprenta Isleña. 
Arredondo y Garcla, Agustín (1875a): Programa de Psicologia, Lógica y Ética. Santa Cruz de Tenerife, Imprenta de J. Benítez y $\mathrm{C}^{2}$, vi +33 páginas.

- (1875b): Compendio doctrinal de Psicologia, Logica y Filosofia Moral. (1. ${ }^{2}$ Parte). Santa Cruz de Tenerife, Imprenta de J. Benítez.

AZNAR GARCIA, José Vicente (1999): La unificación de los pesos y medidas en España durante el siglo XIX. Valencia, Universidad, Tesis Doctoral [Microforma].

Benito y BenITO, Quintín (1876): Memoria acerca del estado del Instituto Provincial de Canarias, leida en la solemne apertura del curso de 1875 á 76 . Santa Cruz de Tenerife, Imprenta de J. Benítez y Compañía.

Cola Benftez, Luis (2001): La Imprenta Benitez. Santa Cruz de Tenerife, C.C.P.C.

Diaz Diaz, G. y Santos Escudero, C. (1982): Bibliografia Filosoffica Hispánica (19011970). Madrid, C.S.I.C. (Instituto de Filosoffa «Luis Vives»).

ESCRIBANO GARRIDO, J. (1987): Los Jesuitas y Canarias, 1566-1767. Granada, Facultad de Teología.

FajARdo SPINOla, F. (1995): Historia del Instituto de Canarias. Santa Cruz de Tenerife, C.C.P.C. y Consejería de Educación, Cultura y Deportes del Gobierno de Canarias.

GLıCK, Thomas F. (1982): Darwin en España. Barcelona, Ed. Península.

Inglott y Navarro, F. (1881): Memoria leida en el Colegio de San Agustín de Las Palmas, en el acto solemne de la apertura del curso académico de 1881 á 1882. Las Palmas, Tip. de I. Miranda.

Lorenzo y GarCfa, Rafael (1869): Programa de las lecciones de Filosofla, (Psicologia, Logica y Filosofia Moral) que se enseñan en el Instituto de Gran-Canaria. Las Palmas, Imp. de la Verdad.

- (1871): Programa de lecciones... Segunda edición. Las Palmas, Imp. de la Verdad.

LUIS Y YAGUE, A. (1874): Memoria leida el $1^{\circ}$ de octubre, en la inauguración del curso académico de 1873 a $1874 \ldots$ en el Instituto de Las Palmas de Gran-Canaria. Las Palmas, Imprenta de la Verdad.

LUXÁN MELÉNDEZ, S. DE (1994): La industria tipográfica en Canarias 1750-1900. Balance de la producción impresa. Las Palmas, Cabildo Insular de Gran Canaria.

MARTIN DEL Castillo, Juan Francisco (1997): «La enseñanza de las ciencias en Canarias durante la segunda mitad del siglo XIX: las matemáticas y las ciencias naturales». Anuario de Estudios Atlánticos, 43, 551-602. 
- (1999): «Libros canarios de matemáticas para la Enseñanza Secundaria (1849-1920)». Epsilon, 43-44, 85-96.

- (2000): «Juan de la Puerta Canseco y la didáctica matemática en Canarias (18571892)». In: Elena Ausejo \& M. ${ }^{2}$ Carmen Beltrán (eds.), La enseñanza de las ciencias: una perspectiva histórica. "Cuadernos de Historia de la Ciencia, 11 ", Zaragoza, Universidad y SEHCTAR, I, pp. 465-474.

Navarro, Domingo J. (1991): Recuerdos de un noventón [1895]. Las Palmas, Cabildo Insular de Gran Canaria.

Negrín FajARdo, Olegario (1982): La enseñanza en Canarias. Col. "La Guagua», Las Palmas de Gran Canaria, Cabildo Insular.

PINTO, J. M. (1870): Memoria leida el dia $1^{\circ}$ de octubre de 1869 con motivo de la solemne apertura de los estudios en el Instituto de $2{ }^{a}$ Enseñanza de Canarias. Santa Cruz de Tenerife, Imprenta Isleña.

- (1871): Memoria leida en dia $1^{\circ}$ de octubre de 1870 con motivo de la solemne apertura de estudios... Santa Cruz de Tenerife, Imprenta Isleña.

ReYMUNDo ARRoyo, Mariano (1879): Discurso leido en el Instituto Provincial de Segunda Enseñanza de Canarias, en el acto solemne de la apertura del curso académico de 1878 a 79 . Santa Cruz de Tenerife, Imprenta de J. Benítez y Compañía.

SÁnchez, J.; Paz, Manuel de (1988): Historia Popular de Canarias. Pensamiento contemporáneo. Santa Cruz de Tenerife, C.C.P.C.

SuÁrez Falcón, J. (1920): Historial de los establecimientos de enseñanza de Las Palmas. Las Palmas, Tip. del «Diario».

Utande Igualada, M. (1964): Planes de estudio de Enseñanza Media (1787-1963). Madrid, Dirección General de Enseñanza Media (MEC).

Vea MuniESA, Fernando (1992): Las matemáticas en la Enseñanza Secundaria en España en el siglo XIX. "Cuadernos de Historia de la Ciencia, 9". Zaragoza, Universidad, 2 vols.

VIERA, Isaac de (1888): Vidas ajenas. Santa Cruz de Tenerife, Imprenta de Hijos de Francisco C. Hernández.

VizCaya CÁRPENTER, A. (1964): Tipografia Canaria. Col. «Bibliografías locales», $n .^{\circ} 2$. Santa Cruz de Tenerife, Instituto de Estudios Canarios. 